Results Women who self-reported infertility had a high prevalence of serologically predicted chlamydial infertility (36\%), which was significantly different from fertile women (18\%). The study accounted for confounders using stepwise multiple logistic regression analysis (BMI, number of cigarettes per day, age). MEDAC CT IgG p-ELISA correlated with self-reported infertility (OR 2.32, 94\% CI 1.25-4.33; P = 0.01), while Anilabsystems CT IgG ELISA correlated with the current infections diagnosed by PCR (OR 1.93, 95\% CI 1.11-3.37; P = 0.02).

Conclusion The study highlights the importance of serological tests in potentially identifying women with CT-related infertility. The high prevalence of CT and women testing positive in CT infertility tests suggests that CT could be a major contributory factor to infertility, and a major unrecognised disease burden in the Samoan population.

Disclosure of interest statement No conflicts of interest.

\section{P08.24 ALTERNATIVE EVIDENCE SOURCES PRESENT A CONSISTENT PICTURE OF THE POPULATION EXCESS FRACTION (PEF) OF PELVIC INFLAMMATORY DISEASE CAUSED BY CHLAMYDIA TRACHOMATIS INFECTION IN ENGLAND}

${ }^{1}$ Malcolm J Price* ${ }^{2}$ AE Ades, ${ }^{3}$ Kate Soldan, ${ }^{3}$ lan Simms, ${ }^{2}$ Nicky J Welton, ${ }^{2}$ John Macleod, ${ }^{4}$ Paddy J Horner. 'School of Health and Population Sciences, University of Birmingham, Birmingham; ${ }^{2}$ School of Social and Community Medicine, University of Bristol, Bristol, UK; ${ }^{3}$ Health Protection Agency, Colindale, London, UK; ${ }^{4}$ Bristol Sexual Health Centre, University Hospital Bristol NHS Foundation Trust, Bristol, UK

\subsection{6/sextrans-2015-052270.370}

Introduction Pelvic Inflammatory Disease (PID) is a leading cause of both Tubal Factor Infertility (TFI) and Ectopic pregnancy. Chlamydia trachomatis (CT) is an important risk factor for the development of PID but it is also caused by other infections. The extent of the role of CT in the aetiology of PID is unclear and knowledge of its role is critical for assessing the efficacy and cost-effectiveness of CT screening.

Methods We define the population attributable fraction as the population Excess fraction (PEF) 'the estimated proportion of the disease that would be removed by eliminating the exposure from the population'. We consider seven separate methods of estimating age-group specific PEFs using a variety of data-sources. Estimates are based on evidence from routine data sources, survey's, case-control studies and randomised controlled trials. Estimation is carried out using a Bayesian approach. This method ensures that all of the uncertainty in the data and estimates for all parameters is fully propagated into the estimates of PEF.

Results There is a high degree of consistency between these estimates of PEF. We estimate that in women aged 16-44 around $20 \%$ of PID is caused by CT in England. However, this could be as low as $5 \%$ or as high as $40 \%$. There is a steep decline in PEF with age with the PEF dropping by a factor of around 5-fold between younger and older women.

Conclusion There is good evidence that CT plays an important role in the development of PID, especially in younger women.
Future studies of the relationship between CT and PID should focus more on the relationship between risk and age.

Disclosure of interest statement This study was funded by the Medical Research Council grant G0801947. No pharmaceutical grants were received in the development of this study. PH has received funding from Cepheid directly and indirectly for lecturing on point of care testing and undertaking research on the cost effectiveness of their CT/NG assay. Has also received payment from Atlas Genetics for an article in the Parliamentary Review on the benefits of point of care technology in improving the cost effectiveness of sexual health services. Has also received an honorarium from Hologic for an education talk on STI diagnostics.

\section{P08.25 TUBAL FACTOR INFERTILITY (TFI) EPIDEMIOLOGY IN INFERTILITY CLINIC PATIENTS}

${ }^{1} \mathrm{RJ}$ Gorwitz*${ }^{*}{ }^{2} \mathrm{KR}$ Hammond, ${ }^{3,4} \mathrm{HC}$ Wiesenfeld, ${ }^{5} \mathrm{NA}$ Cataldo, ${ }^{1} \mathrm{KA}$ Sereday, ${ }^{1} \mathrm{DM}$ Kissin, ${ }^{3} \mathrm{~F}$ Balmir, ${ }^{4,6} \mathrm{CL}$ Haggerty, ${ }^{5} \mathrm{EW}$ Hook, ${ }^{2} \mathrm{MP}$ Steinkampf, ${ }^{1} \mathrm{~L}$ Markowitz, ${ }^{5} \mathrm{WM}$ Geisler. ${ }^{1}$ United States Centers for Disease Control and Prevention; ${ }^{2}$ Alabama Fertility Specialists; ${ }^{3}$ University of Pittsburgh Medical Center; ${ }^{4}$ Magee-Women's Research Institute; ${ }^{5}$ University of Alabama Medical Center; ${ }^{6}$ University of Pittsburgh, Graduate School of Public Health

\subsection{6/sextrans-2015-052270.371}

Introduction Prevention of tubal factor infertility (TFI) is a primary objective of chlamydia prevention programs. This study aimed to describe TFI epidemiology in infertility clinic patients. Methods Data were analysed from one site of an ongoing twosite study. Medical record data from infertile (unable to conceive for $\geq 12$ months, no history of tubal sterilisation) women aged 19-42 yrs with an initial consultation at a private infertility practice in Birmingham, Alabama during 01/01/2011-06/30/2012 were abstracted to a standardised form. TFI was defined by report of fallopian tube occlusion on hysterosalpingogram, hydrosalpinx on pelvic ultrasound, and/or evidence of fallopian tube damage (e.g., tubal occlusion, hydrosalpinx, peritubal adhesions) on laparoscopy. Statistical tests were used to compare proportions (Fisher's exact, chi-square) and medians (Wilcoxon).

Results Eligible patients $(\mathrm{N}=413$, median age $31 \mathrm{yrs}$ ) included 87 black, 303 white and 23 other race women, who had been trying to conceive a median of 30 months (48 months black vs 24 months white, $\mathrm{p}<0.001)$ at initial consultation. Recorded history of chlamydia and pelvic inflammatory disease (PID) were more common in black than white patients $(9.2 \%$ vs $1.3 \%, \mathrm{p}=$ $0.001 ; 6.9 \%$ vs $1.6 \%, \mathrm{p}=0.02)$. TFI was identified in 82 (19.9\%) women $(32.2 \%$ black vs $15.8 \%$ white, $\mathrm{p}<0.001)$. Among 82 women with and 331 without TFI, a history of chlamydia was recorded in $2.4 \%$ vs $3.3 \%(\mathrm{p}=1.0)$, gonorrhoea in $0 \%$ vs $1.2 \%(\mathrm{p}=1.0)$, PID in $6.1 \%$ vs $1.8 \%(\mathrm{p}=0.047)$, and ectopic pregnancy in $14.6 \%$ vs $2.4 \%$ ( $p<0.001$ ).

Conclusion TFI was identified in one-fifth of infertility patients. Black women had been attempting to conceive longer before initial consultation than white women, and had a higher prevalence of TFI. Known sexually transmitted infection (STI) history was uncommon, but more prevalent in blacks. Studies using biological measures of exposure (e.g., serology) are needed to better define the proportion of TFI attributable to STIs. 